\title{
Primer registro de Cooperia curticei (Strongylida: Trichostrongylidae) en un ovino de la región del noroeste argentino
}

\author{
Olmos $\mathrm{LH}^{1,2^{*}}$, Colque Caro LA ${ }^{1,2,3}$, Avellaneda-Cáceres $\mathrm{A}^{2,3}$, Aguirre $\mathrm{LS}^{2,3}$, \\ Micheloud $\mathrm{JF}^{1,2,3}$, Suarez $\mathrm{VH}^{1}$ \\ 1 Instituto Nacional de Tecnología Agropecuaria (INTA), -CIAP -Instituto de Investigación Animal del \\ Chaco Semiárido -Área de Investigación en Salud Animal, Estación Experimental Agropecuaria (EEA) \\ Salta (Cerrillos), Argentina. \\ ${ }^{2}$ Facultad de Ciencias Agrarias y Veterinarias, Universidad Católica de Salta, Argentina. \\ ${ }^{3}$ Consejo Nacional de Investigaciones Científicas y Técnicas (CONICET), Argentina.
}

* Correspondencia: Leandro Olmos, INTA EEA Salta. Ruta Nacional 68 km 172, 4403 Cerrillos, Salta, Argentina. E-mail: olmos.leandro@inta.gob.ar

Recibido: 21 Marzo 2020. Aceptado: 22 Junio 2021. Disponible en línea: 12 Julio 2021

Editor: P. Beldomenico

RESUMEN. Cooperia curticei, es un nematodo afín a los ovinos que se aloja en el intestino delgado de estos. Su presencia en Argentina ha sido registrada en ambientes como la región semiárida en la Pampa, subhúmeda pampeana en Buenos Aires y en la región subtropical en Corrientes. El objetivo de este trabajo es notificar la presencia de Cooperia curticei en intestino delgado de un ovino proveniente de la localidad de Cerrillos en Salta. En el intestino delgado se determinó una carga de 600 formas adultas de Cooperia spp. Estas fueron identificadas como Cooperia curticei. Como características taxonómicas de relevancia las ramas del rayo dorsal se curvaban hacia atrás mostrando una forma de lira. En cuanto a las espículas, midieron en promedio $146,8 \mu m( \pm 5,24)$, con una concavidad marcada en parte media del cuerpo y con un tallo con una curvatura final similar a un pie. Este hallazgo constituye el primero en su tipo en la región del NOA y demuestra la adaptación de $C$. curticei a un clima de tipo serrano sub-andino. Este trabajo complementa lo descripto previamente en la región, en donde se menciona la presencia de Cooperia spp en ovinos, sin especificar su especie.

SUMMARY. First report of Cooperia curticei (Strongylida: Trichostrongylidae) in sheep from northwestern Argentina. Cooperia curticei is a nematode related to sheep that is housed in the small intestine. Its presence in Argentina has been reported in environments such as the semi-arid region in the Pampa, the sub-humid Pampas in Buenos Aires and in the subtropical region in Corrientes. The objective of this work is to report the presence of Cooperia curticei in the small intestine of a sheep from the town of Cerrillos in Salta. A worm burden of 600 adult forms of Cooperia spp. was determined in the small intestine. These were identified as Cooperia curticei. As relevant taxonomic features the branches of the dorsal ray curved backwards showing a lyre shape. As for the spicules, they measured an average of $146.8 \mu \mathrm{m}( \pm 5.24)$, with a marked concavity in the middle part of the body and with a stem with a final curvature similar to a foot. This finding is the first of its kind in the NOA region and demonstrates the adaptation of $C$. curticei to a sub-Andean mountain type climate. This work complement that previously described in the region, where the presence of Cooperia spp. in sheep was described but without species determination.

Palabras clave: Nematode; Cooperia; Ovinos; Salta

Keywords: Nematode; Cooperia; Ovine; Salta

Cooperia curticei, es un nematodo afín a los ovinos que se aloja en el intestino delgado de estos (Suarez, 2007). También ha sido identificado en otros rumiantes como caprinos (Sissay et al., 2007) y cérvidos (Amarante et al., 1997). Normalmente se lo considera como una especie de baja patogenicidad y escaso impacto en la salud de los ovinos (Ahluwalia y Charleston, 1975) aunque la información al respecto es limitada.
En Argentina, este parásito ha sido informado previamente en ovinos por Suarez et al., (1994). Ellos describieron la presencia de $C$. curticei al este de la región semiárida pampeana en una baja carga y principalmente en asociación a sistemas extensivos que basan su oferta forrajera en pastizales naturales. Por otro lado, otros trabajos la mencionan como género prevalente en ensayos de eficacia antihelmíntica en la 
región del noreste argentino (Fiel et al., 2011) y en la región Sub Húmeda Pampeana (Steffan et al., 2011; Muchiut et al., 2019).

El objetivo de este trabajo es notificar la presencia, y describir morfológicamente, el hallazgo de Cooperia curticei en intestino delgado de un ovino (Ovis aries) cruza Hampshire $x$ Texel, proveniente de la localidad de Cerrillos, provincia de Salta. Siendo este el primer reporte de dicha especie de Cooperia en la región del NOA.

En febrero de 2021 el Servicio de Diagnóstico Veterinario Especializado INTA Salta, realizó una visita a un establecimiento de cría ovina ubicado en la localidad de Cerrillos, provincia de Salta. Dicha localidad se encuentra ubicada en el Valle de Lerma, el cual posee un clima de tipo serrano sub-andino, con una marcada estación seca que se prolonga desde mayo hasta noviembre y una estación húmeda que se extiende desde diciembre hasta abril con precipitaciones promedios de unos 400 a $900 \mathrm{~mm}$ anuales (Bianchi y Bravo, 2008)

El motivo de la visita fue por la muerte súbita de una oveja adulta sin signología previa aparente. El animal fue procesado mediante la técnica de necropsia con el fin de diagnosticar la causa de su deceso. Durante el procesamiento del animal se decidió enviar al laboratorio de parasitología del Área de investigación animal (AISA-Salta) del Instituto de Investigación Animal del Chaco Semiárido (IIACS) el tracto gastrointestinal para realizar el conteo de parásitos adultos según la técnica descripta por Suárez (1997). Siguiendo dicha técnica, el abomaso y el intestino delgado fueron abiertos y lavados con agua corriente depositando en un balde el agua del lavado del contenido digestivo. Posteriormente, una alícuota del $10 \%$ de este lavado, de cada órgano, fue observada en lupa y los especímenes encontrados fueron recolectados para su visualización en microscopio óptico e identificación morfológica en base a las características taxonómicas descriptas por Skryabin (1991). En el caso del estudio morfológico de los especímenes de Cooperia spp., previamente fueron aclarados en lactofenol por 24 horas y para su identificación taxonómica de especie se utilizaron las características descriptas por Gibbons (1980).

A la necropsia el animal presentaba lesiones relacionadas a un cuadro de emaciación progresiva como digestión serosa de las grasas, ascitis e hidropericardio. A su vez, también se observó palidez de las mucosas aparentes, lo cual indicaría que el animal presentaba un cuadro de anemia severa.

En cuanto a los recuentos de adultos se observó una carga alta de Haemonchus contortus (4100 adultos y 900 inmaduros) a nivel abomasal y en el intestino delgado se contabilizaron 1200 formas adultas de Trichostrongylus columbriformis y 600 formas adultas de Cooperia spp. Estas últimas, en el estudio morfo- lógico fueron identificados como Cooperia curticei. Dicha caracterización de especie estuvo basada en las características observadas en el estudio de 15 machos. Estos tuvieron una longitud promedio de $5,83 \mathrm{~mm}$ ( \pm $0,317)$, y como características taxonómicas de relevancia no presentaban papilas prebursales y en el rayo dorsal las ramas se curvaban hacia atrás mostrando una forma de lira (Figura 1A). En cuanto a las espículas, la longitud promedio de estas fue de $146,8 \mu \mathrm{m}( \pm 5,24)$, con una concavidad marcada en parte media del cuerpo y con un tallo con una curvatura final similar a un pie (Figura 1B). Por último, no se observó la presencia de gubernaculum.

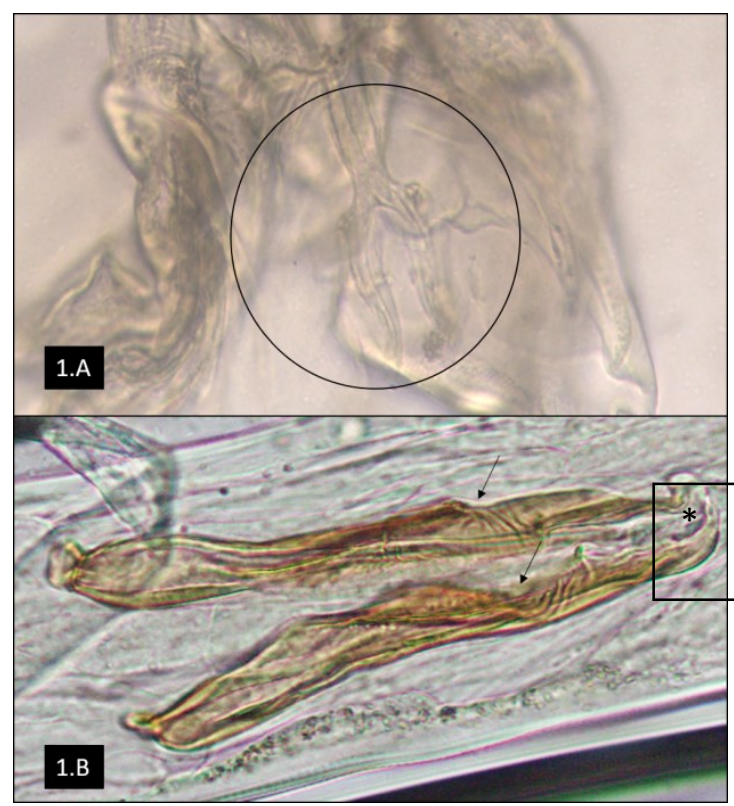

Figura 1. (1.A) Rayo dorsal con forma de lira (círculo). 1B. Concavidad cerca del cuerpo medio de la espícula (flecha) y terminación del tallo de la espícula con forma de pie (asterisco).

Según el estudio morfológico de los especímenes analizados, se pudo llegar a la conclusión que se trataba de Cooperia curticei. La morfología descripta coincide con lo observado por otros autores en cuanto a las formas (Ransom, 1911; Gibbons, 1981). Sin embargo, tomando en cuenta la medición de la longitud de las espículas, lo observado en este trabajo discrepa con lo mencionado por otros autores. Gibbons (1981), determinó un rango de longitud de 165 a $212 \mu \mathrm{m}$ para las mismas, mientras que Ransom (1911), describe un rango de 135 a $145 \mu \mathrm{m}$. En este estudio esta longitud fue de 135,2 a 160,8 $\mu \mathrm{m}$. Dichas diferencias podrían estar debidas a la adaptación del parásito a los distintos ambientes en donde fueron identificados, ya que, en el caso de Gibbons (1981) el estudio se efectuó sobre especímenes provenientes del continente africano y Ransom (1911) de Norteamérica.

En Argentina, aunque la información disponible es escasa, $C$. curticei parece tener la capacidad de adaptarse a diferentes climas. Esto puede afirmarse ya que esta especie ha sido observada en climas templado- 
húmedos (Steffan et al., 2011; Muchiut et al., 2019), en clima templado semiárido (Suarez et al., 1994) y en climas subtropicales (Fiel et al., 2011). Nuestro hallazgo constituye el primero en su tipo en la región del NOA y demuestra la adaptación de $C$. curticei a un clima de tipo serrano sub-andino. Esta información es complementaria a la descripta por Künhe (1986), quien describe la presencia de Cooperia spp. en ovinos $\sin$ precisar la especie.

La descripción de las distintas especies de nematodos gastrointestinales nos permite obtener información de relevancia en el complemento del estudio de su dinámica ambiental en las distintas regiones del país. Dicha información es importante para establecer programas de control sustentables contra los parásitos que generan impacto productivo en los establecimientos ganaderos.

\section{Referencias}

Ahluwalia JS, Charleston WAG. 1975. Studies on the pathogenicity of Cooperia curticei for sheep. NZ. Vet. J. 23: 197-199.

Amarante AFT, Bagnola J, Amarante MRV, Barbosa MA. 1997. Host specificity of sheep and cattle nematodes in Sao Paulo state, Brazil. Vet. Parasitol. 73: 89-104.

Bianchi AR, Bravo GC. 2008. Ecorregión Norandina: Descripción, subregiones, agroecosistemas, sistemas productivos y cartografía regional. INTA EEA Salta, Ediciones INTA. 60 pp.

Fiel C, Guzmán M, Steffan P, Rodríguez E, Prieto O, Bhushan C. 2011. The efficacy of trichlorphon and naphthalophos against multiple anthelmintic-resistant nematodes of naturally infected sheep in Argentina. Parasitol. Res. 109: 139-148.

Gibbons LM. 1981. Revision of the African species of the genus Cooperia Ransom, 1907 (Nematoda, Trichostrongylidae). Syst. Parasitol. 2: 219-252.

Kühne GI. 1986. Parásitos diagnosticados en el decenio 1976 1985 en la Unidad regional de Investigación en Sanidad Animal del noroeste Argentino. I. Helmintos y protozoarios. RIA, INTA, Argentina. 1: 73-78.

Muchiut SM, Fernández AS, Lloberas M, Steffan PE, Luque SE, Cardozo PA, Fiel CA. 2019. Recovery of fenbendazole efficacy on resistant Haemonchus contortus by management of parasite refugia and population replacement. Vet. Parasitol. 271: 31-37.

Ransom BH. 1911. The nematodes parasitic in the alimentary tract of cattle, sheep, and other ruminants. US Government Printing Office. 127: 70-71

Skryabin KI, Shikhobalova NP, Mougovoi AA., 1991. In: Brill, E.J. (Ed.), Key to parasitic nematodes. Volume 2: Oxyurata and Ascaridata. K“'oln, Leiden, New York, Kobenhaven, pp. 530-533.

Sissay MM, Uggla A, Waller PJ. 2007. Prevalence and seasonal incidence of nematode parasites and fluke infections of sheep and goats in eastern Ethiopia. Trop. Anim. Health Prod. 39: 521-531.

Steffan P, Sánchez E, Entrocasso C, Fiel C, Lloberas M, Riva E, Guzmán M. 2011. Eficacia de monepantel contra nematodes de ovinos con resistencia antihelmíntica múltiple en la Región Templada de Argentina. Vet. Arg. 273: 12-16.

Suárez VH. 1997. Diagnóstico de las parasitosis internas de los rumiantes en la región de invernada. Técnicas e Interpretación. Bol. Divul. Tec. (INTA-Anguil) 56: 50.

Suárez V.H. 2007. Epidemiología y control de los nematodos gastrointestinales en el oeste de la Región Pampeada. In: Suarez, V.H., Olaechea, F.V., Rossanigo, C.E., Romero, J.R. (Eds), Enfermedades parasitarias de los ovinos y otros rumiantes menores en el Cono Sur de América, Ediciones INTA, Anguil, Argentina, PT. Pp. 43-62.

Suárez VH. Busetti MR, Bedotti OO, Fort MC. 1994. Parasitosis internas de los ovinos en la Prov. De La Pampa Semiárida. Rev. Fac. Agron. 7: 35-42.

Salvador A, Martínez G. 2007. Factores que Afectan la Producción y Composición de la Leche de Cabra: Revisión Bibliográfica Rev. Fac. Cs. Vet. UCV. 48: 61-76.

SIGSA. 2017. Dirección de Control de Gestión y Programas Especiales - Dirección Nacional de Sanidad Animal - SENASA. [Online] (https://www.argentina.gob.ar/senasa/caprinossector-primario).

Soares Filho G, McManus C, Mariante AS. 2001. Fatores genéticos e ambientais que influenciam algumas características de reprodução e produção de leite em cabras no Distrito Federal. Rev. Bras. Zootecnia 30: 133-140.

Suárez VH, Martínez GM. 2019. Características de los tambos caprinos comerciales y posibilidades de mejora genética en el noroeste argentino. Revista Veterinaria Argentina 36: 1-13.

Takma C, Akbaş Y, Taskin T. 2009. Modeling lactation curves of Turkish Saanen and Bornova goats. Asian J. Anim. Vet. Advances 4: 122-129.

Torres-Vázquez JA, Valencia-Posadas $M$, Castillo-Juárez $H$, Montaldo HH. 2010. Genetic and phenotypic trends for milk yield and milk composition traits of Saanen goats from Mexico, Rev. Mex. Cien. Pecuarias 1: 337-348.

Zamuner F, DiGiacomo K, Cameron AWN, Leury BJ. 2020. Effects of month of kidding, parity number, and litter size on milk yield of commercial dairy goats in Australia. J. Dairy Sci. 103: 954-964. 